\title{
Current Barriers in Y-Ba-Cu-O Coated Conductors
}

\author{
J. L. Reeves, D. M. Feldmann, C-Y. Yang, \& D. C. Larbalestier
}

\begin{abstract}
Current barriers in high temperature superconductors occur on many length scales. Eliminating them is critical to making superconductors more favorable for commercial applications. In state-of-the-art $\mathrm{YBa}_{2} \mathrm{Cu}_{3} \mathrm{O}$ (YBCO) coated conductors, barriers to current in short lengths of tapes are grain boundaries. On the scale of several tens of micrometers, the role of grain boundaries in the nickel substrate was investigated using a combination of plan-view scanning electron microscopy (SEM), Backscattered Electron Kikuchi Pattern (BEKP) analysis, magneto-optical (MO) imaging, and Focused Ion Beam (FIB) analysis. The combined techniques showed enhanced magnetic flux penetration, and hence reduced critical current density $\left(J_{c}\right)$, in the YBCO above nickel grain boundaries that have misorientation angles $(\theta)$ greater than $4^{\circ}$ in pulsed laser deposited $\mathrm{YBCO}$ films and $5^{\circ}$ in $\mathrm{BaF}_{2}$-grown YBCO films. Additionally, BEKP percolation maps show that the YBCO layer is single-crystal-like below a percolation limit of a few degrees; the BEKP grain boundary maps displays the constrictions of the current path on the scale of 50-100 $\mu \mathrm{m}$. On the sub-micrometer scale, the misorientation angles of grain boundaries in the YBCO layer were investigated using transmission electron microscopy imaging and diffraction analysis. The average and range of $\theta$ between neighboring YBCO islands above a single grain of the metal substrate were essentially the same in two samples with $J_{c}$ values that differed by a factor of four. This suggests that defects on the macroscale, specifically the underlying nickel grain boundaries, may influence the $J_{c}$ more than defects on the microscale. The results of this study suggest that that the way to improve the superconducting properties in the YBCO is to eliminate $\theta>4-5^{\circ}$ boundaries in the nickel substrate or engineer the conductors in a way to increase the threshold angle.
\end{abstract}

Index Terms - RABiTS, grain boundary, orientational imaging, magneto-optical imaging, YBCO microstructure

\section{INTRODUCTION}

$\mathrm{C}$ oated conductor technology makes it feasible to produce long lengths of $\mathrm{YBa}_{2} \mathrm{Cu}_{3} \mathrm{O}$ (YBCO) with a highly textured microstructure. The general structure of coated conductors is a metallic substrate (nickel or nickel alloy) on which buffer layers (typically a combination of YSZ and

Manuscript received September 18, 2000. This work was supported by AFOSR, DOE, EPRI and the NSF-funded MRSEC on Nanostructured Materials.

J. L. Reeves, D. M. Feldmann, C-Y. Yang, \& D. C. Larbalestier are with the Applied Superconductivity Center, University of Wisconsin-Madison, Madison, WI 53706 (telephone: 608-263-2194, e-mail: larbales@engr.wisc.edu).
$\mathrm{CeO}_{2}$ ) and $\mathrm{YBCO}$ are grown [1]. In the case of the Rolling Assisted Biaxially Textured Substrate (RABiTS) method, a high degree of crystallographic texture is produced in the metal substrate [2] and transferred to the YBCO through epitaxial growth, reducing the density of high angle grain boundaries in the superconductor. Eliminating high angle grain boundaries is important because bicrystal studies have shown that the critical current $\left(\mathrm{J}_{\mathrm{c}}\right)$ is strongly dependent on the grain boundary misorientation angle. Dimos et al. [3] and Heinig et al. [4] showed that the $J_{c}$ across a grain boundary is reduced to a small fraction of the intragrain $J_{c}$ if the angle of misorientation $(\theta)$ is $>7^{\circ}$. This weak link behavior makes it necessary for long lengths of YBCO to contain few or no higher angle grain boundaries in order to optimize $J_{c}$ for commercial applications.

This study of RABiTS-type coated conductors explores the microstructure of the YBCO and its relationship to $J_{c}$ on two length scales: the $50-100 \mu \mathrm{m}$ grain size in the nickel substrate and the sub-micrometer island size of the YBCO. It shows that the bottlenecks in the current path of currently available RABiTS-type coated conductors are associated with grain boundaries in the underlying nickel.

\section{EXPERIMENTAL}

Spatial variations in $\mathrm{J}_{\mathrm{c}}$ and the misorientation angles of grain boundaries in the same region of the nickel substrate were investigated by magneto-optical (MO) imaging and backscattered electron Kikuchi pattern (BEKP) analysis, respectively [5]. Experiments were conducted on coated conductors with pulsed laser deposited (PLD) YBCO obtained from the Air Force Research Laboratory and on coated conductors with $\mathrm{BaF}_{2}$-deposited $\mathrm{YBCO}$ obtained from Oak Ridge National Laboratory. Both coated conductors had the same sequence of buffer layers: $\mathrm{Ni} / \mathrm{CeO}_{2} / \mathrm{YSZ} / \mathrm{CeO}_{2} / \mathrm{YBCO}$. MO images obtained under standard imaging conditions $[6,7]$ were used to locate the areas of reduced $J_{\mathrm{c}}$ in the YBCO layer. These images were correlated with grain boundary misorientation maps that were deduced from BEKPs obtained from the same area of the nickel substrate. The BEKP data was collected in a field emission scanning electron microscope (SEM); the sample was tilted at $70^{\circ}$ and the voltage was $20 \mathrm{kV}$. The beam was rastered over a roughly $500 \times 500 \mu \mathrm{m}$ area with $3 \mu \mathrm{m}$ step size.

To investigate the structure-property relationship on the sub-micrometer scale, transmission electron microscopy (TEM) was used to measure the misorientation angles $(\theta)$ between PLD YBCO islands in two different 


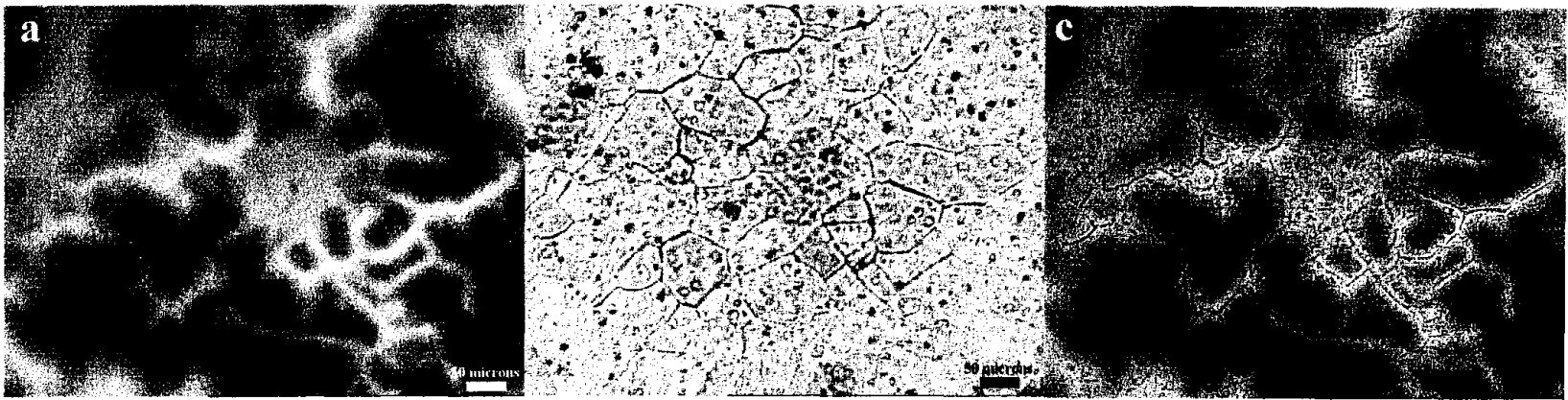

Fig. 1. Images of PLD YBCO coated conductor surface: (a) MO image; (b) light microscope image of YBCO surface; (c) overlay MO and light microscope images to show that many, but not all, boundaries are visible in MO.

$\mathrm{Ni} / \mathrm{YSZ} / \mathrm{CeO}_{2} / \mathrm{YBCO}$ tapes. The $\mathrm{J}_{\mathrm{c}}$ values of the two tapes differed by more than a factor of four $\left(3 \mathrm{MA} / \mathrm{cm}^{2}\right.$ and 0.7 $\left.\mathrm{MA} / \mathrm{cm}^{2}\right)[8,9]$.

\section{RESULTS AND DISCUSSION}

The MO image of a sample with $300 \mathrm{~nm}$ thick PLD YBCO is shown in Fig. 1a. Dark areas are regions that are electromagnetically well-connected. Bright areas indicate magnetic flux penetration and areas of locally depressed $J_{\mathfrak{c}}$. The most striking features in the MO image are regions surrounded by bright lines; these regions range in diameter from $50-100 \mu \mathrm{m}$. Fig. $1 \mathrm{~b}$ shows the light microscopy image of the surface of the YBCO. Fig. 1c shows the overlay of the MO image and the light microscopy image. The overlay suggests that many, but not all, of the boundaries visible in the light microscope image of the YBCO correspond to bright lines in the MO image. In addition, most of the boundaries that are bright in MO images are also visible in plan-view SEM images of PLD YBCO (Fig. 2a). To a lesser extent, Fig. $2 \mathrm{~b}$ shows that these boundaries are also visible in $\mathrm{BaF}_{2}$ YBCO with relatively high $J_{\mathfrak{c}}\left(2.3 \mathrm{MA} / \mathrm{cm}^{2}\right)$.

To determine what was causing the macroscale boundaries in the YBCO, a Focused Ion Beam (FIB) was used to cut trenches across boundaries that appeared in the YBCO layer. The nickel grain boundaries are visible in the bottom of the FIB trenches (Fig. 3), giving direct evidence that the

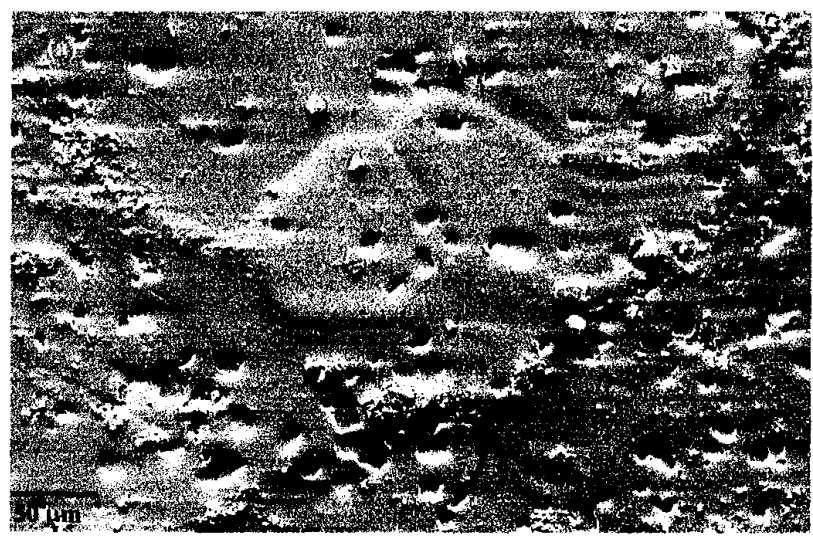

boundaries seen in the YBCO layer appear directly above grain boundaries in the nickel substrate.

Individual Kikuchi patterns were obtained from smooth regions on the $300 \mathrm{~nm}$ thick PLD YBCO surface using a very low noise CCD camera with long acquisition times. Fig. 4 shows the Kikuchi patterns from adjacent grains in a region of the sample where one of the boundaries between the grains was visible as a bright line in the MO image. To illustrate the grain boundary misorientation in the YBCO layer, the triangle formed by the intersection of bands in the Kikuchi pattern was traced in area B in Fig. 4. Comparing the rotation of the Kikuchi pattern relative to the fixed triangle position shows that there is a larger misorientation, specifically 4.2 degrees, between grains $\mathrm{A}$ and $\mathrm{B}$ than between grains $\mathrm{B}$ and $\mathrm{C}$, which were misoriented by only 0.7 degrees. Thus, the brighter boundary in the MO image corresponds to a higher misorientation angle between grains $\mathrm{A}$ and $\mathrm{B}$ than the boundary between $\mathrm{B}$ and $\mathrm{C}$.

To summarize, combined BEKP analysis and $\mathrm{MO}$ imaging show that the boundaries between YBCO regions that are misoriented by more than $4^{\circ}$ have locally reduced $J_{c}$. FIB trenches cut through all the buffer layers at the position of the grain boundary grooves in the YBCO confirm that these boundaries appear directly over grain boundaries in the nickel substrate to within the 1 micrometer resolution of the technique.

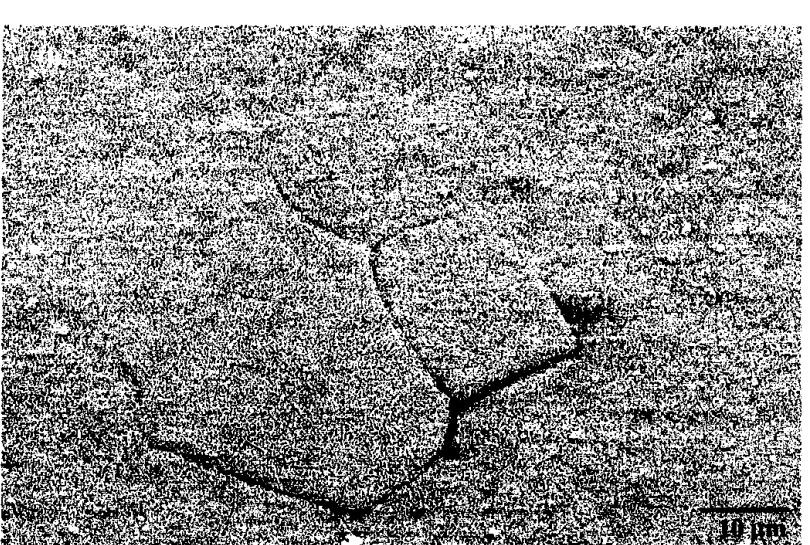

Fig. 2, Plan-view SEM images of (a) PLD YBCO and (b) BaF -grown YBCO on RABiTS-type substrates. Bright areas in MO images often correspond to boundaries that are visible in the SEM. 


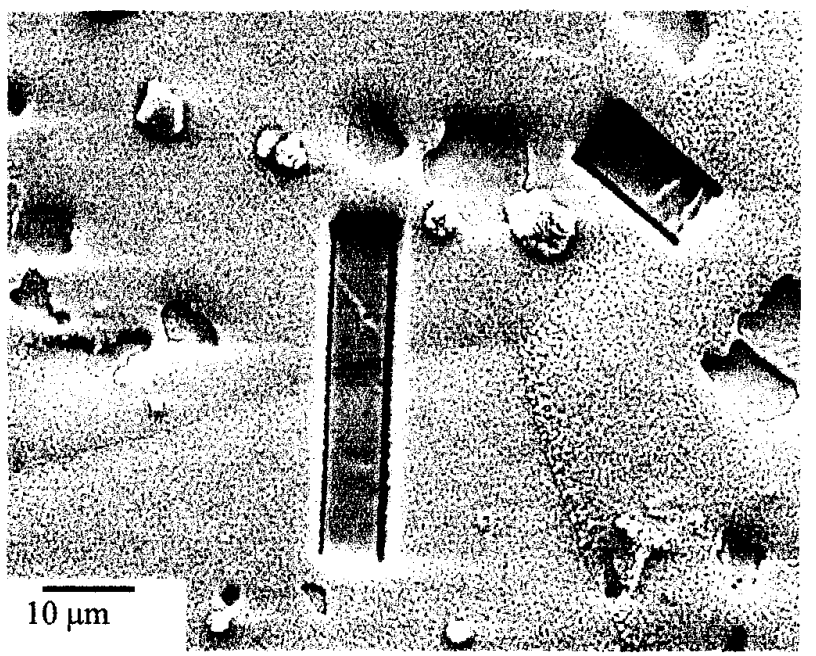

Fig. 3. SEM image of PLD YBCO surface. Two trenches were cut with the FIB to directly observe the underlying nickel substrate.

After the $1.2 \mu \mathrm{m}$ thick PLD YBCO and buffer layers were removed by chemical etching, BEKP analysis was done on a large region of the nickel substrate. A grain orientation map is shown in Fig. 5a. In the literature [10], this type of map is called a "percolation map" in which a grain is defined as a set of points for which each point has a neighbor that has the same crystallographic orientation, in this case to within $1^{\circ}$. Each collection of points with neighbors within $1^{\circ}$ of each other is shaded a different color in the map. The $1^{\circ}$ percolation map correlates well to the light microscope image of the nickel substrate. Similarly, Fig. $5 \mathrm{~b}$ is a percolation map for which the crystal orientation criterion is $4^{\circ}$. This $4^{\circ}$

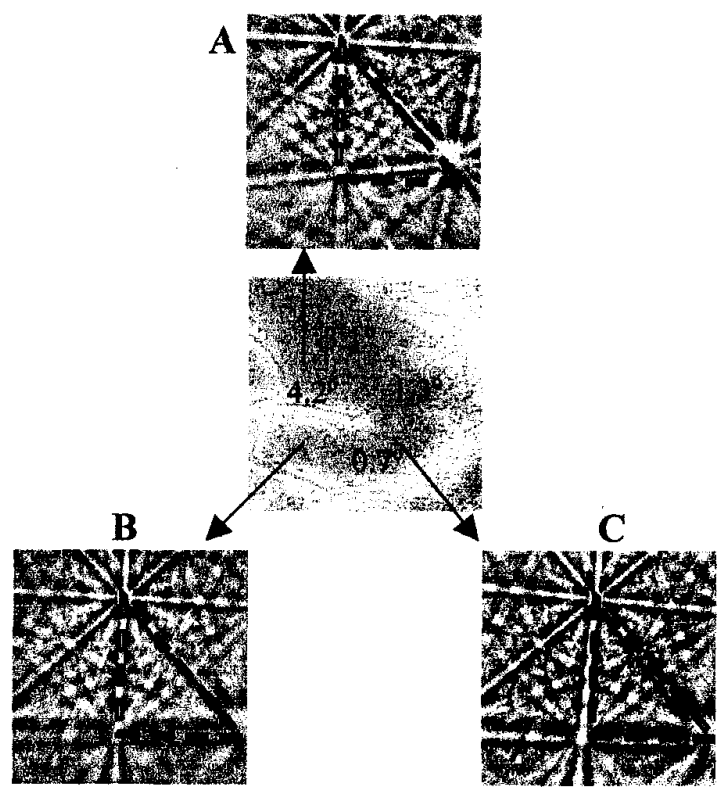

Fig. 4. Representative Kikuchi patterns from three areas on the PLD YBCO surface. The amount of misalignment is qualitatively shown by the change in position of the Kikuchi pattern (as denoted by the dashed triangle). The misorientation is labeled in the MO image.

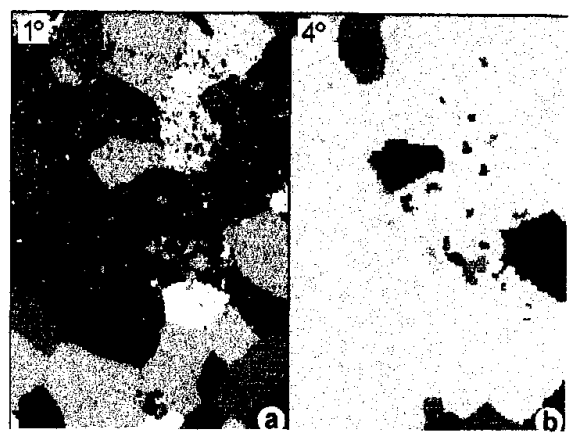

Fig. 5. Percolation maps from BEKP data where the grain-tograin orientation criterion is $1^{\circ}$ in (a) and $4^{\circ}$ in (b).

percolation map might suggest that the majority of the sample is single-crystal-like with respect to current flow, a conclusion that does not agree with the many bright regions visible in the MO image.

Fig. 6 is a collection of "grain boundary maps." Each map shows the location of all grain boundaries for which the grain boundary misorientation angle, $\theta$, is greater than the value indicated on the image, in this case from $2^{\circ}$ through $7^{\circ}$. Fig. 7 is a comparison of the $\theta>4^{\circ}$ grain boundary map, the $4^{\circ}$ percolation map, and the MO image. Several high angle grain boundaries that are visible in the grain boundary map and $\mathrm{MO}$ images are not evident in the percolation map. This comparison indicates that percolation maps do not show all barriers to current flow. In addition, there is an almost one-toone correlation between the location of boundaries with $\theta>$ $4^{\circ}$ and the flux penetration network visible in the MO image. This comparison indicates that the YBCO that grows above nickel grain boundaries with $\theta>4^{\circ}$ has reduced $J_{c}$.

The difference between percolation maps and grain boundary maps is important. Percolation maps can be used to make the point that the YBCO layer is single-crystal-like below a percolation limit of a few degrees. While monochromatic percolation maps imply that there is a fully connected path through the microstructure that is within the chosen angle criterion of the map, it is the grain boundary map that displays the constrictions of the current path. Thus it is the grain boundary map and its correlation to the MO image that confirms the role of the nickel grain boundaries in the constriction of the current flow.

In this $1.2 \mu \mathrm{m}$ thick PLD YBCO sample, we correlated nickel grain boundary misorientation angle and MO image intensity. The MO images were taken when the sample was in its critical state; in other words, further increasing the magnetic field did not lead to any more grain boundaries becoming bright in the MO image. The magnitude of flux penetration in the YBCO above more than 60 of the nickel grain boundaries was estimated by measuring the MO image intensity, which is roughly proportional to the penetrating magnetic field. Plotting the MO image intensity vs. nickel grain boundary misorientation angle in Fig. 8 shows that there is an abrupt change in MO intensity at $4^{\circ}$ : magnetic flux does not preferentially penetrate grain boundaries with $\theta<4^{\circ}$, while boundaries with $\theta>4^{\circ}$ do exhibit MO contrast. Above the $4^{\circ}$ 


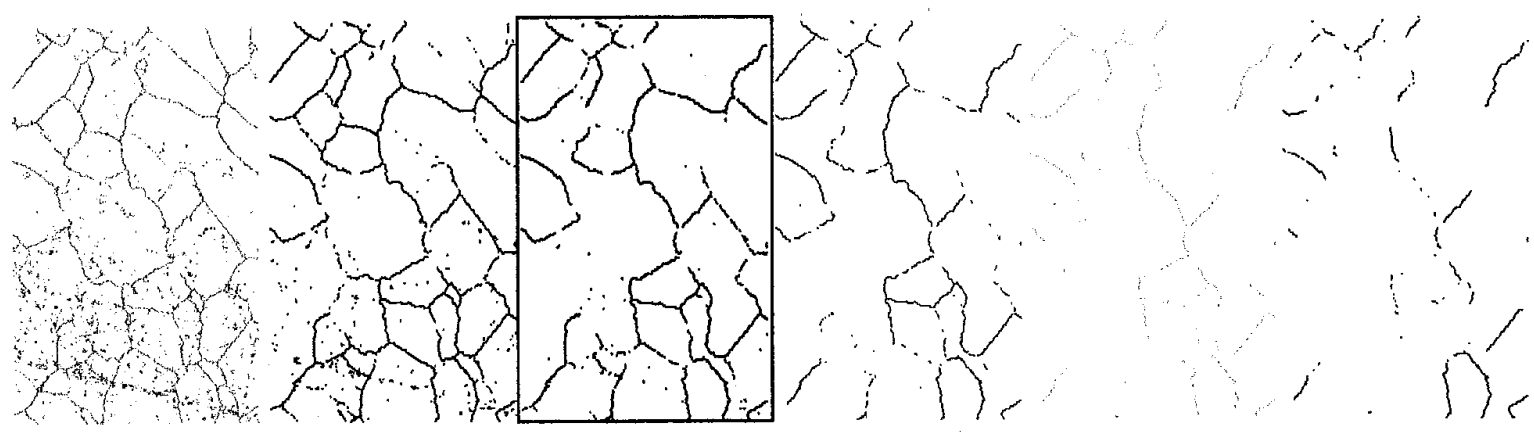

Fig. 6. Grain boundary maps. The boundary criteria is $2,3,4,5,6,7^{\circ}$ from left to right, with the $\theta>4^{\circ}$ grain boundary map outlined for emphasis. The $\theta>2^{\circ}$ map shows the most barriers. The $\theta>7^{\circ}$ map has many fewer barriers than the $\theta>4^{\circ}$ map.

threshold angle, the magnitude of flux penetration varies considerably and not very systematically with $\theta$. The presence of a sharp threshold angle at $4^{\circ}$ suggests that one way to improve $\mathrm{J}_{\mathrm{c}}$ in coated conductors is to make nickel substrates where all of the grain boundaries have $\theta<4^{\circ}$. Another improvement to coated conductors could be made if it was possible to engineer an increased threshold angle. This may be possible, since YBCO bicrystal data $[4,11]$ shows a threshold angle that is as high as $7^{\circ}$ in some cases.

This experiment was repeated to determine a correlation between $\theta$ and $\mathrm{MO}$ image intensity of $\mathrm{BaF}_{2}$-grown $\mathrm{YBCO}$ on the RABiTS substrate for which the overall $J_{c}$ was $2.3 \mathrm{MA} / \mathrm{cm}^{2}$. In these samples, the threshold angle was approximately $5^{\circ}$; in other words, the YBCO above nickel grain boundaries with $\theta<5^{\circ}$ could carry as much current as the bulk YBCO.

On the sub-micrometer scale, TEM was used to investigate the properties of the YBCO that grew above the nickel grain interiors. Fig. 9 shows diffraction contrast TEM images of PLD YBCO with $J_{c}=3 \mathrm{MA} / \mathrm{cm}^{2}$. Superimposed on the TEM image are local $\theta$ measurements. Most of the boundaries have $\theta<3^{\circ}$, but the $\theta$ values range from nearly $0^{\circ}$ up to $7^{\circ}$ for a small number of boundaries. In most regions, the range of measured $\theta$ 's was very small, but some regions showed a larger $\theta$ range over areas of a few square micrometers, indicating that the highly misoriented islands tend to form in

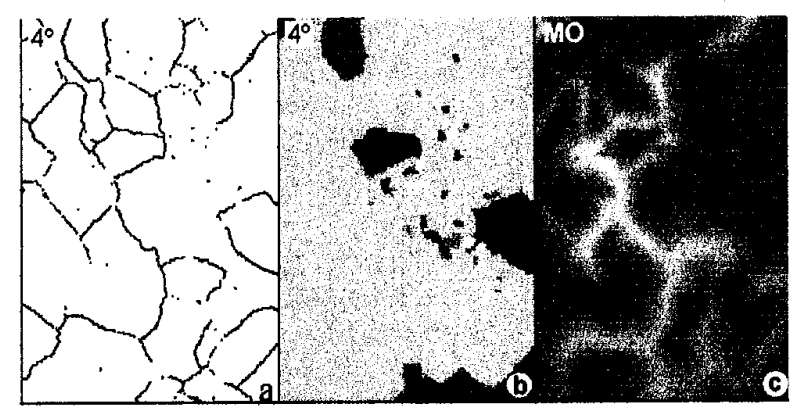

Fig. 7. Comparison of (a) BEKP $\theta>4^{\circ}$ grain boundary map, (b) $4^{\circ}$ percolation map, and (c) MO image (c) that shows a good correlation between the grain boundary map and the MO image. clusters. Histograms of the distribution of $\theta^{\prime}$ s and the average value of $\theta\left(3^{\circ}\right)$ were essentially the same in both the very high $J_{c}$ and the lower $J_{c}$ samples. This interesting result suggests that microstructural features other than the local island-toisland $\theta$ control the $J_{c}$ value, even for very good RABiTS tapes. Given that $J_{c}$ is defined only as $I_{c} / A$, it is in fact much more likely that the differences in $\mathrm{J}_{\mathrm{c}}$ are determined more by variations in the active cross-section seen on the MO-visible scale $(50-100 \mu \mathrm{m})$ than by the much smaller (submicrometer) flux pinning scale.

\section{CONCLUSION}

Using a combination of SEM imaging, FIB analysis, MO imaging, and BEKP analysis, we have shown that there are macroscale barriers to current flow in the YBCO layer of conventional RABiTS-type coated conductors due to grain boundaries in the nickel that influence the connectivity of the YBCO grains. BEKP percolation maps show that the YBCO layer is single-crystal-like below a percolation limit of a few degrees, while the BEKP grain boundary maps displays the constrictions of the current path on the scale of $50-100 \mu \mathrm{m}$. On the sub-micrometer scale, there is measurable mosaic spread among the YBCO islands of PLD coated conductors, but the mosaic spread does not seem to affect $J_{c}$. We conclude that $J_{c}$ may be increased by improving the biaxial texture in the underlying metal substrate.

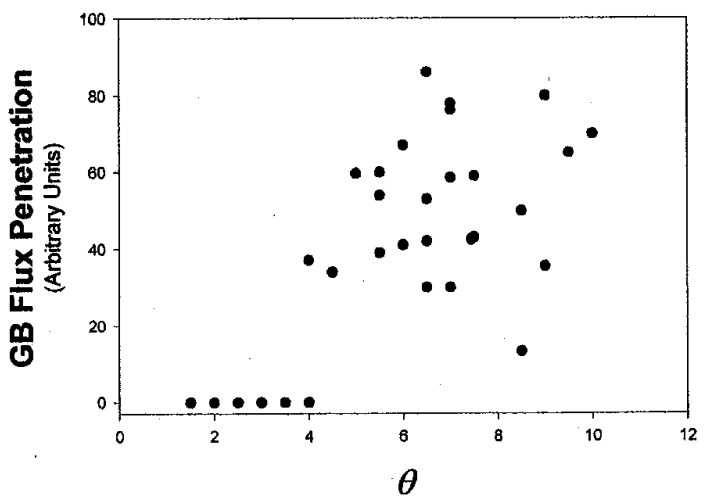

Fig. 8. Plot of MO image intensity vs. grain boundary misorientation angle. Bright areas in the MO image correspond to nickel grain boundaries with $\theta>4^{\circ}[5]$. 


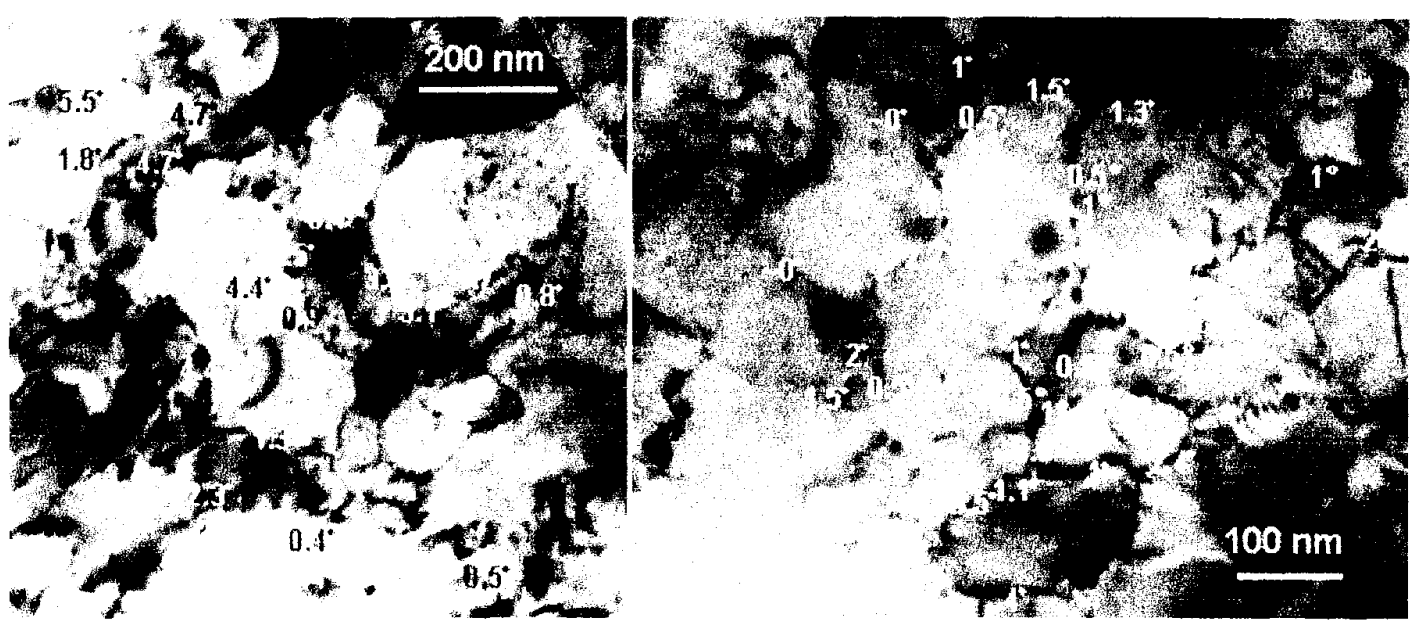

Fig. 9. TEM images overlaid with local $\theta$ measurements for two different areas in a high $\mathrm{J}_{\mathrm{c}}\left(3 \mathrm{MA} / \mathrm{cm}^{2}\right)$ PLD YBCO sample. The $\theta$ values for the area shown in the left image range from less than $1^{\circ}$ to more than $5^{\circ}$, while most $\theta^{\prime} \mathrm{s}$ are $<2^{\circ}$ in the right image.

\section{ACKNOWLEDGMENT}

We are grateful to colleagues at the Air Force Research Laboratory (T.L. Peterson, R.M. Nekkanti, R.R. Biggers, G. Kozlowski, I. Maartense, P. Barnes, C.E. Oberly,), Oak Ridge National Laboratory (A. Goyal, R. Feenstra, M. Paranthaman, D.M. Kroeger, D.K. Christen), and Los Alamos National Laboratory (Steve Foltyn) who have supplied us with samples for on-going studies. We are also grateful to P. Camus and D. Rohde at Noran Instruments, Middleton, WI, for BEKP technical assistance.

\section{REFERENCES}

[1] D. Norton, "Science and technology of high-temperature superconducting films," Ann. Rev. Mat. Sci., vol. 28, pp. 299-343, 1998 , and references contained therein.

[2] R. Feenstra, T. B. Lindemer, J. D. Budai and M. D. Galloway, "Effect of oxygen pressure on the synthesis of $\mathrm{YBa}_{2} \mathrm{Cu}_{3} \mathrm{O}_{7 \times \mathrm{x}}$ thin films by postdeposition annealing," J. Appl. Phys., vol. 69, pp. 6569-6585, 1991.

[3] D. Dimos, P. Chaudhari and J. Mannhart, "Superconducting transport properties of grain boundaries in $\mathrm{YBa}_{2} \mathrm{Cu}_{3} \mathrm{O}_{7}$," Phys. Rev. B, vol. 41, pp. 4038-4049, 1990.

[4] N. Heinig, R. D. Redwing, I. F. Tsu, A. Gurevich, J. E. Nordman, S. E. Babcock and D. C. Larbalestier, "Evidence for channel conduction in low misorientation angle [001] tilt $\mathrm{YBa}_{2} \mathrm{Cu}_{3} \mathrm{O}_{7 \times x}$ bicrystal films, "Appl. Phys. Lett., vol. 69, pp. 577-579, 1996.

[5] D. M. Feldmann, J. L. Reeves, A. A. Polyanskii, G. Kozlowski, R. R. Biggers, R. M. Nekkanti, I. Maartense, M. Tomsic, P. Barnes, C. E. Oberly, T. L. Peterson, S. E. Babcock and D. C. Larbalestier, "Influence of nickel substrate grain structure on $\mathrm{YBa}_{2} \mathrm{Cu}_{3} \mathrm{O}_{7-\mathrm{x}}$ supercurrent connectivity in deformation-textured coated conductors," $A P L$, vol. 77, pp. 2906-2908, 2000.

[6] A. A. Polyanskii, A. Gurevich, A. E. Pashitski, N. F. Heinig, R. D. Redwing, J. E. Nordman and D. C. Larbalestier, "Magneto-optical study of flux penetration and critical current densities in s001]tilt $\mathrm{YBa}_{2} \mathrm{Cu}_{3} \mathrm{O}_{7-\delta}$ thin-film bicrystals," Phys. Rev. B, vol. 53, pp. 8687. $8697,1996$.

[7] A. E. Pashitski, A. Gurevich, A. A. Polyanskii, D. C. Larbalestier, A. Goyal, E. D. Specht, D. M. Kroeger, J. A. DeLuca and J. E. Tkaczyk, "Reconstruction of current flow and imaging of current-limiting defects in polycrystalline superconducting films," Science, vol. 275, pp. 367$369,1997$.

[8] A. E. Pashitski, A. Gurevich, A. A. Polyanskii, D. C. Larbalestier, A. Goyal, E. D. Specht, D. M. Kroeger, J. A. DeLuca and J. E. Tkaczyk, "Reconstruction of current flow and imaging of current-limiting defects in polycrystalline superconducting films," Science, vol. 275, pp. 367$369,1997$.

[9] F. A. List, F. A. List, A. Goyal, M. Paranthaman, D. P. Norton, E. D. Specht, D. F. Lee and D. M. Kroeger, "High $J_{c}$ YBCO films on biaxially textured $\mathrm{Ni}$ with oxide buffer layers deposited using electron beam evaporation and sputtering," Physica $C$, vol. 302, pp. 87-92, 1998.

[10] J. E. Mathis, A. Goyal, D. F. Lee, F. A. List, M. Paranthaman, D. K. Christen, E. D. Specht, D. M. Kroeger and P. M. Martin, "Biaxially textured $\mathrm{YBa}_{2} \mathrm{Cu}_{3} \mathrm{O}_{7-\delta}$ conductors on rolling assisted biaxially textured substrates with critical current densities on $2.3 \mathrm{~mA} / \mathrm{cm}^{2}$," Jap. J. Appl. Phys., vol. 37, pp. L1379-1382, 1998.

[11] A. Goyal, S. X. Ren, E. D. Specht, D. M. Kroeger, R. Feenstra, D. Norton, M. Paranthaman, D. F. Lee and D. K. Christen, "Texture formation and grain boundary networks in rolling assisted biaxially textured substrates and in epitaxial YBCO films on such substrates," Micron, vol. 30, pp. 463-478, 1999.

[12] N. F. Heinig, R. D. Redwing, J. E. Nordman and D. C. Larbalestier, "Strong to weak coupling transition in low misorientation angle thin film $\mathrm{YBa}_{2} \mathrm{Cu}_{3} \mathrm{O}_{7}-$ x bicrystals," Phys. Rev. $B$, vol. 60, pp. 1409-1417, 1999. 